Supporting Information

\title{
A Scalable Cathode Chemical Prelithiation Strategy for Advanced Silicon-based Lithium Ion Full Batteries
}

Zongzhe Liu ${ }^{1, \#}$, Shaobo Ma ${ }^{1, \#}$, Xue Mu${ }^{1}$, Renlong $\mathrm{Li}^{1}$, Geping Yin ${ }^{1}$, Pengjian Zuo ${ }^{1, *}$

1 MITT Key Laboratory of Critical Materials Technology for New Energy Conversion and Storage, School of Chemistry and Chemical Engineering, Harbin Institute of Technology, Harbin 150001, China 
(a)

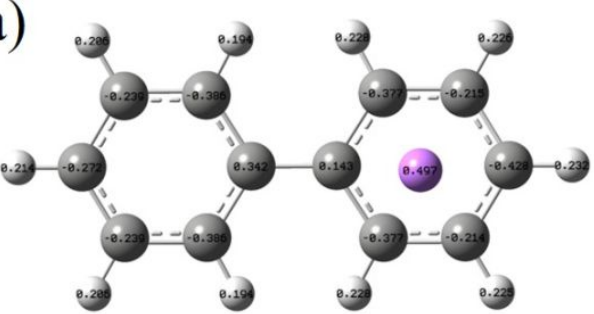

Charge distribution

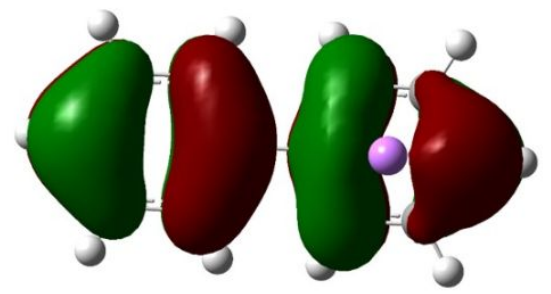

HOMO

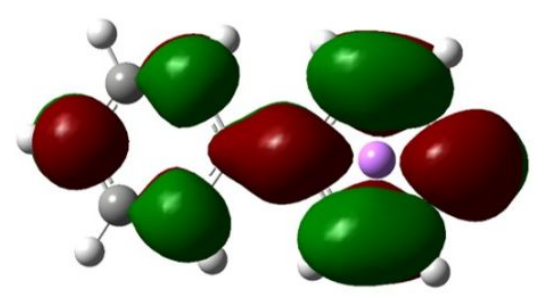

LUMO (b)

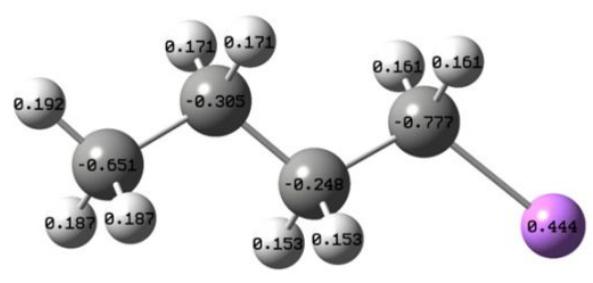

Charge distribution

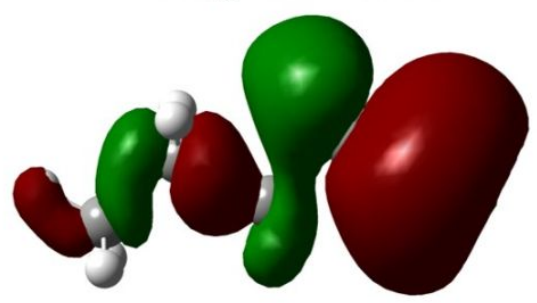

HOMO

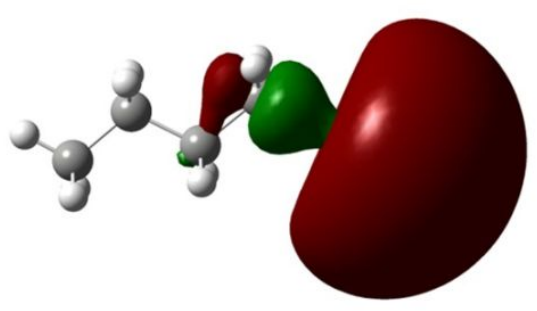

LUMO

Figure S1. Quantum calculation including charge distribution, HOMO and LUMO orbital distribution of (a) Li-biphenyl and (b) butyl lithium. 

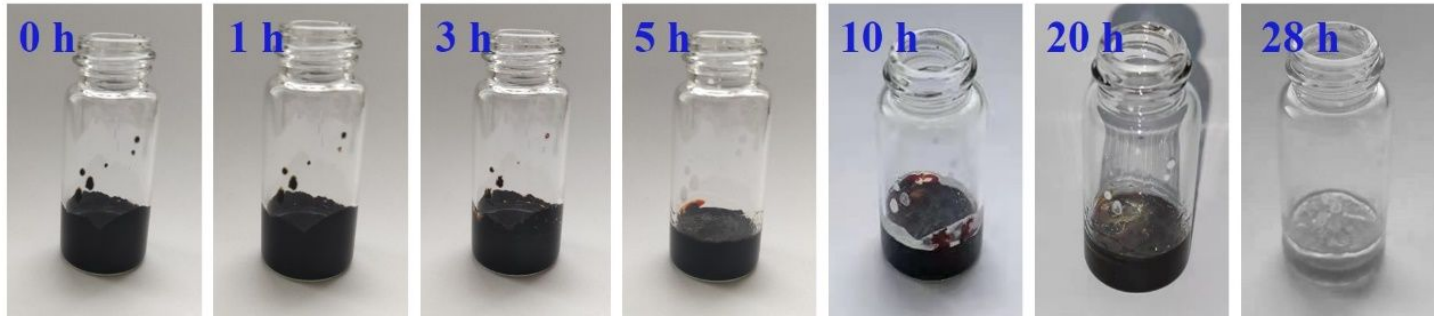

Figure S2. The inactivation process of Li-BP when exposed to open air in $28 \mathrm{~h}$.

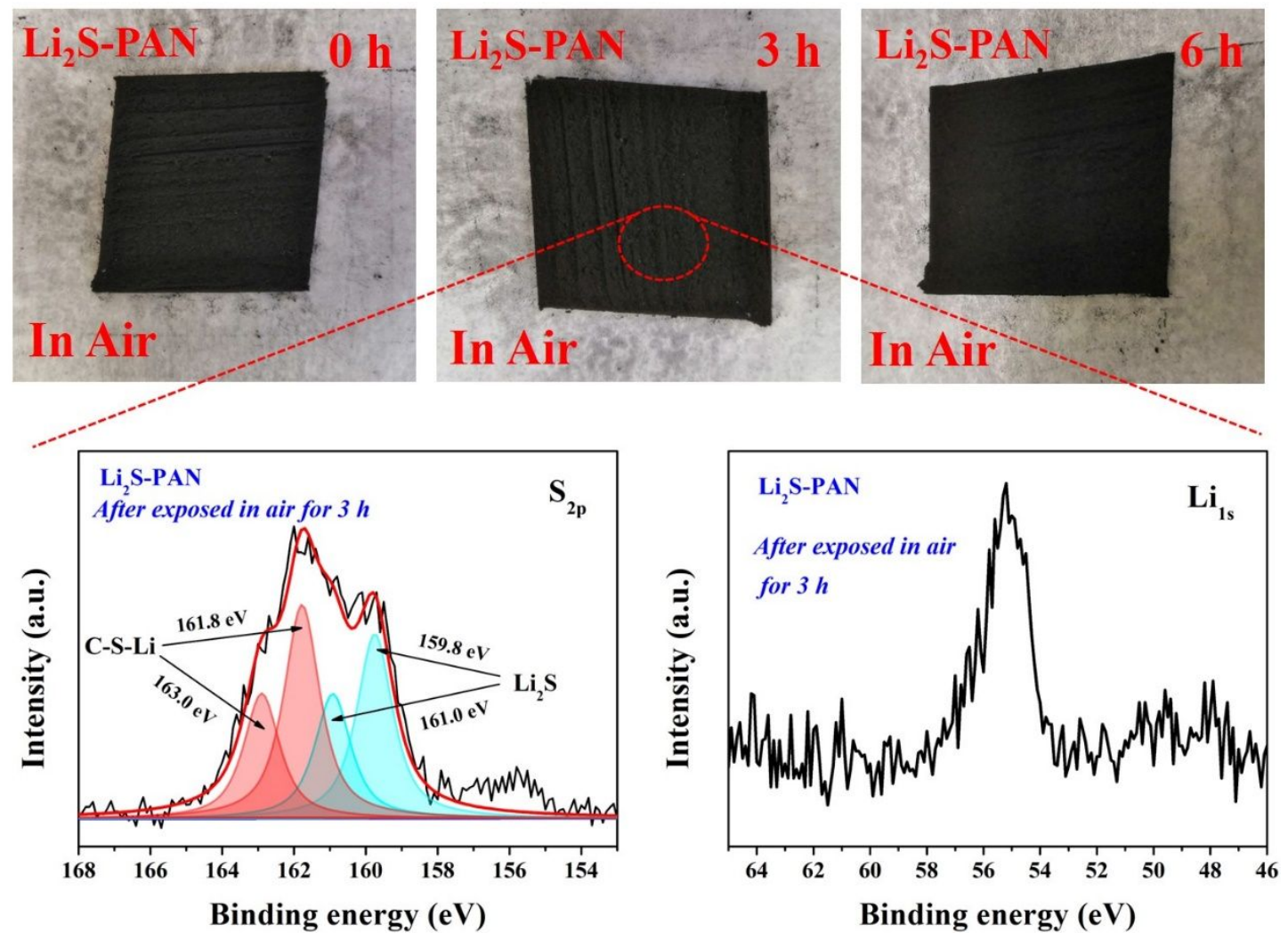

Figure S3. The air stability of $\mathrm{Li}_{2} \mathrm{~S}-\mathrm{PAN}$ powder when exposed in open air for $6 \mathrm{~h}$. 


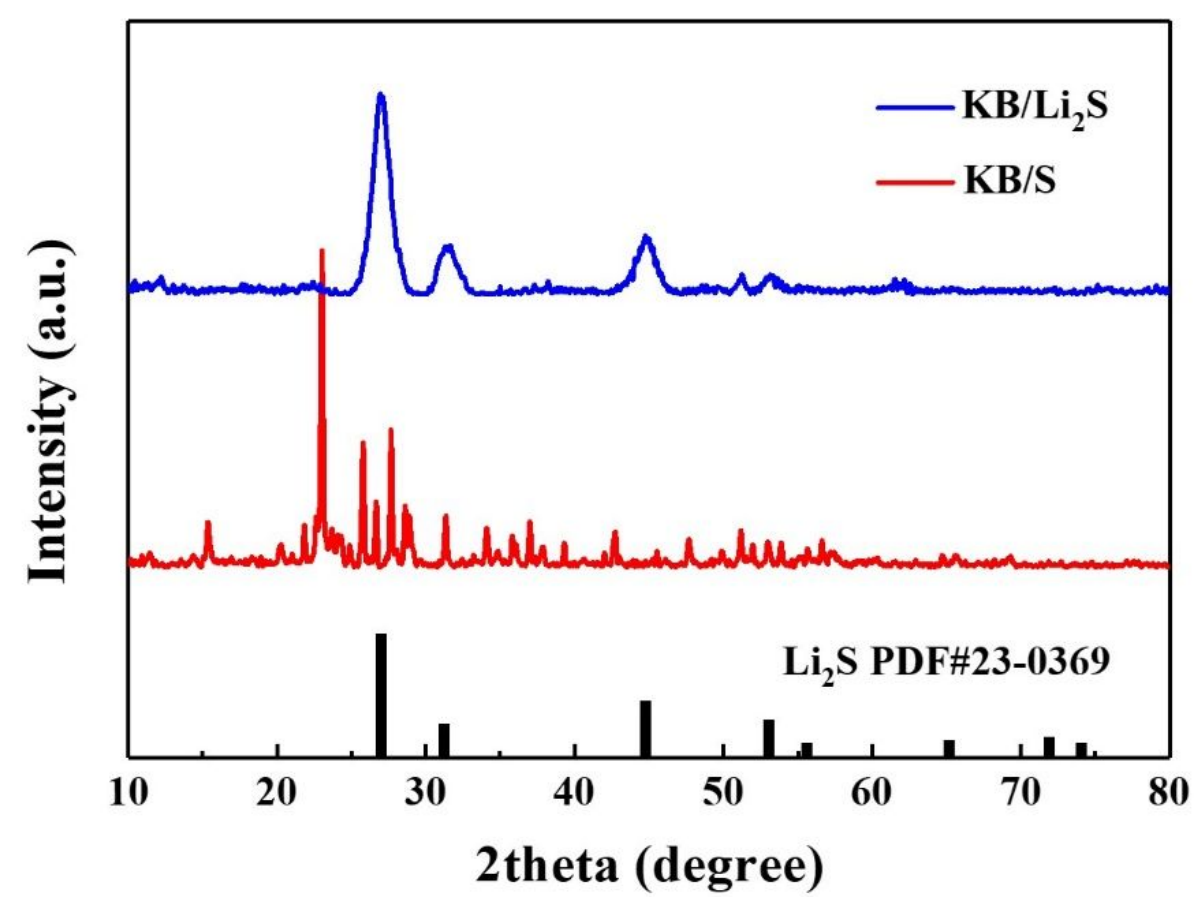

Figure S4. XRD pattern of KB/S material before and after chemical prelithiation.

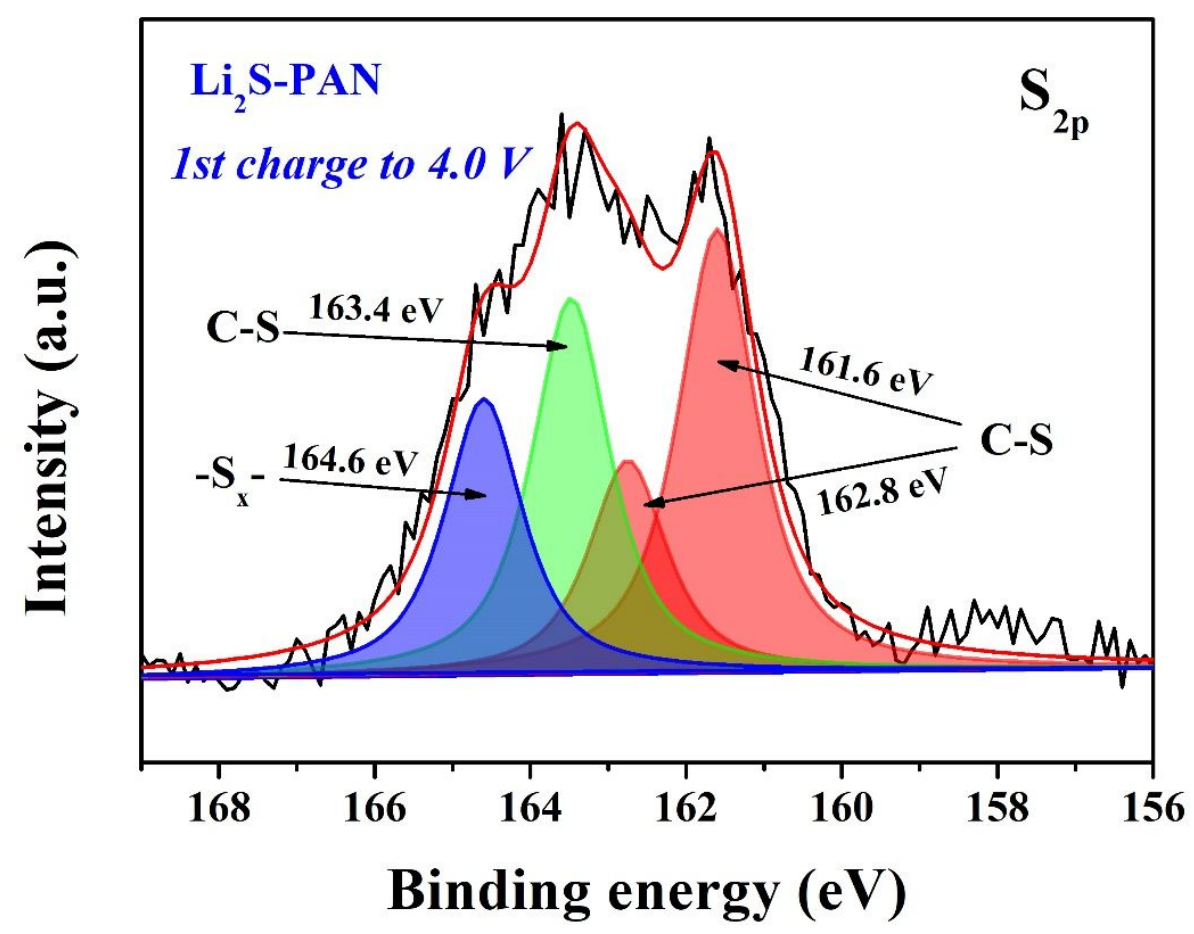

Figure S5. $\mathrm{S}_{2 \mathrm{p}} \mathrm{XPS}$ spectrum of $\mathrm{Li}_{2} \mathrm{~S}-\mathrm{PAN}$ after first charging to $4.0 \mathrm{~V}$. 


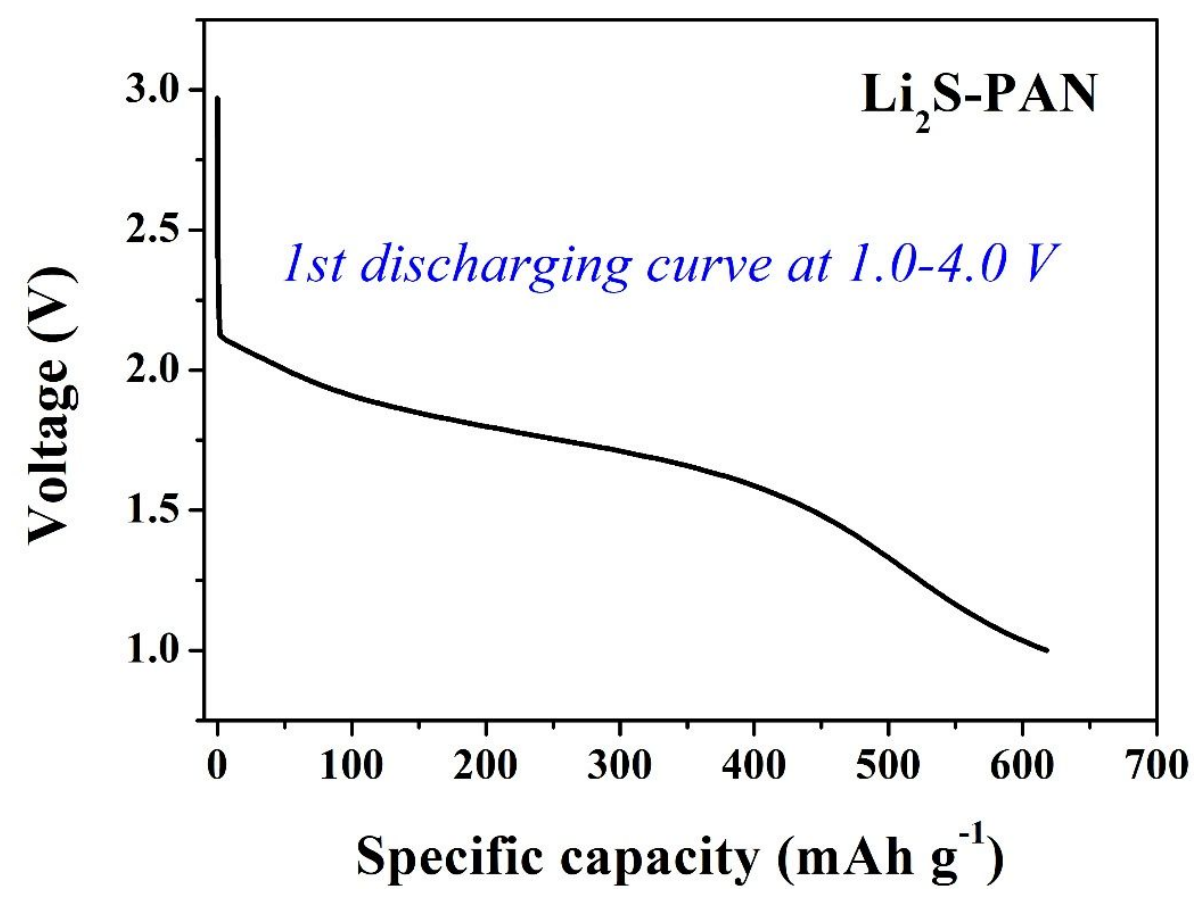

Figure S6. The initial discharging curve of $\mathrm{Li}_{2} \mathrm{~S}-\mathrm{PAN}$ at a voltage of 1.0-4.0 V.

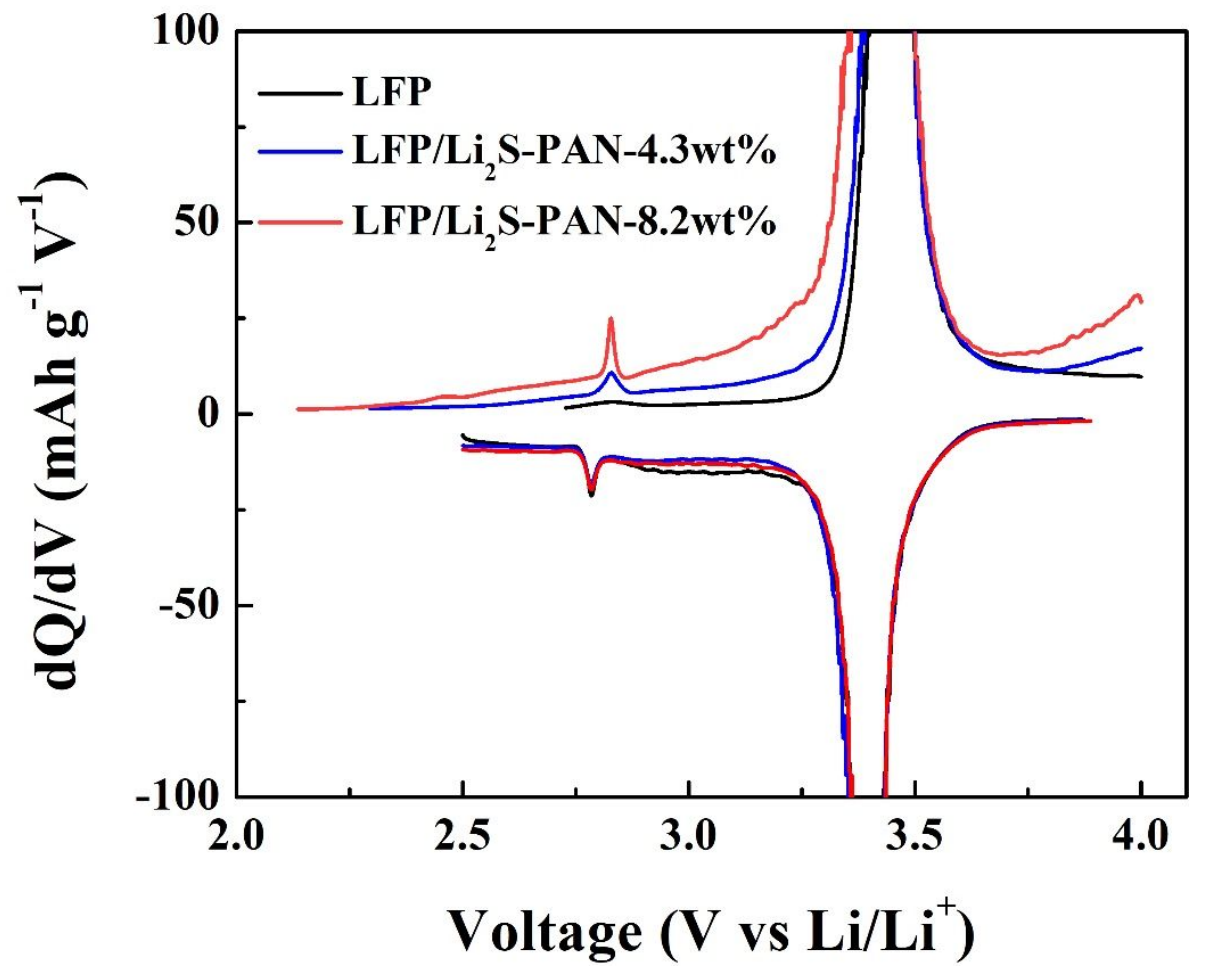

Figure S7. Differential capacity curve of first cycle of LFP, LFP/Li $2 \mathrm{~S}-\mathrm{PAN}-4.3 \mathrm{wt} \%$ and $\mathrm{LFP} / \mathrm{Li}_{2} \mathrm{~S}-\mathrm{PAN}-8.2 \mathrm{wt} \%$. 


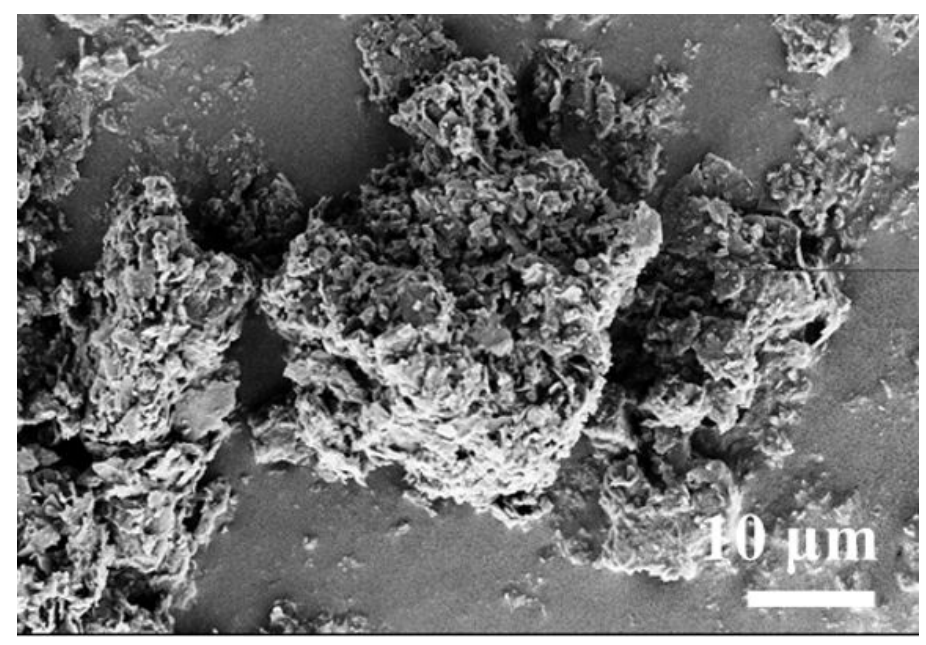

Figure S8. The SEM images of Si/G/C composite.
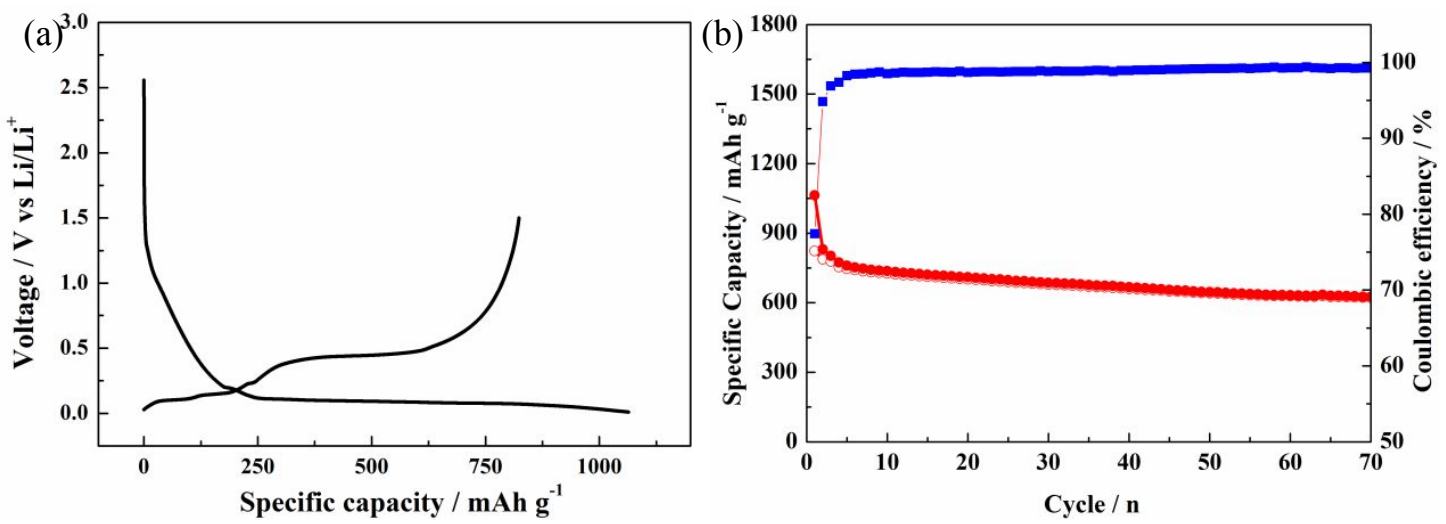

Figure S9. Electrochemical properties of $\mathrm{Si} / \mathrm{G} / \mathrm{C}$ composite, (a) initial charge/discharge curve and

(b) cycle performance. 


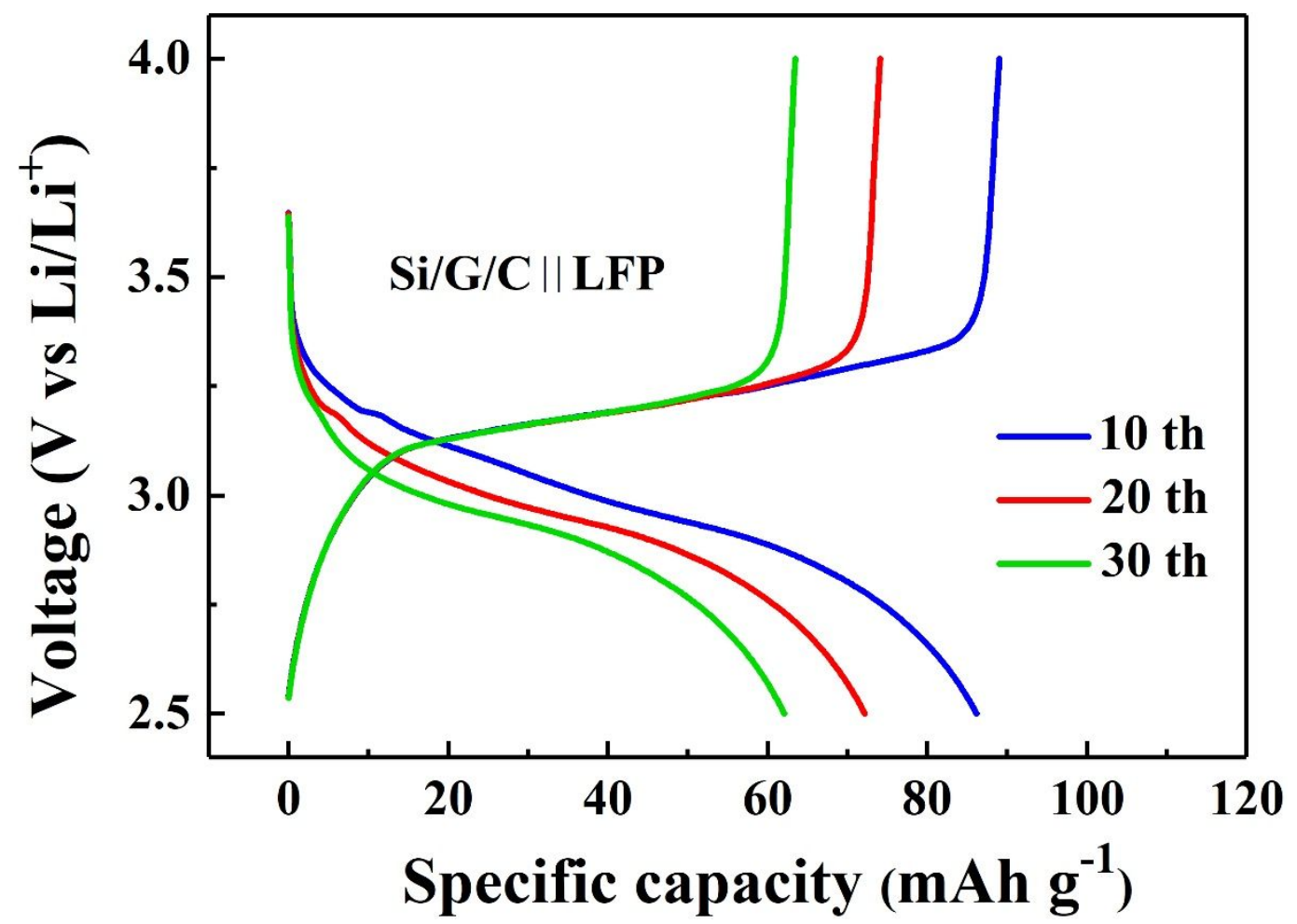

Figure S10. The (dis-)charge curves of $\mathrm{Si} / \mathrm{G} / \mathrm{C} \| \mathrm{LiFePO}_{4}$ full-cell at various cycles.

Table S1. Comparison of electrochemical properties and preparation methods of previously reported cathode prelithiation materials.

\begin{tabular}{|c|c|c|c|c|}
\hline Materials & Preparation method & $\begin{array}{c}\text { Work } \\
\text { potential }\end{array}$ & $\begin{array}{l}\text { Irreversible } \\
\text { capacity }\end{array}$ & Reference \\
\hline $\mathrm{Li}_{5} \mathrm{FeO}_{4}$ & $\begin{array}{l}\text { High-temperature } \\
\text { sintering }\end{array}$ & $2.75-4.5 \mathrm{~V}$ & $665 \mathrm{mAh} \mathrm{g}^{-1}$ & {$[1]$} \\
\hline $\mathrm{Fe} / \mathrm{LiF} / \mathrm{Li}_{2} \mathrm{O}$ & $\begin{array}{l}\text { High-temperature } \\
\text { sintering }\end{array}$ & $2.5-4.4 \mathrm{~V}$ & $550 \mathrm{mAh} \mathrm{g}^{-1}$ & {$[2]$} \\
\hline $\mathrm{Li}_{2} \mathrm{O}_{2} / \mathrm{NCM}$ & Ball milled & $2.8-4.6 \mathrm{~V}$ & $1154 \mathrm{mAh} \mathrm{g}^{-1}$ & {$[3]$} \\
\hline $\mathrm{Li}_{3} \mathrm{~N}$ & $\begin{array}{l}\text { Calcined after nitriding } \\
\text { in a glove box }\end{array}$ & $\begin{array}{l}4.8 \mathrm{~V} \\
4.5 \mathrm{~V}\end{array}$ & $\begin{array}{l}1761 \mathrm{mAh} \mathrm{g}^{-1} \\
1163 \mathrm{mAh} \mathrm{g}^{-1}\end{array}$ & {$[4]$} \\
\hline $\mathrm{Co} / \mathrm{Li}_{2} \mathrm{O}$ & Sintering & $2.5-4.1 \mathrm{~V}$ & $609 \mathrm{mAh} \mathrm{g}^{-1}$ & {$[5]$} \\
\hline LiDHBA & Liquid phase reaction & $2.0-4.3 \mathrm{~V}$ & $301.6 \mathrm{mAh} \mathrm{g}^{-1}$ & {$[6]$} \\
\hline $\mathbf{L i}_{2} \mathbf{S}-\mathbf{P A N}$ & Liquid phase reaction & 2.5-4.0 V & $668.1 \mathrm{mAh} \mathrm{g}^{-1}$ & This work \\
\hline
\end{tabular}


(a)

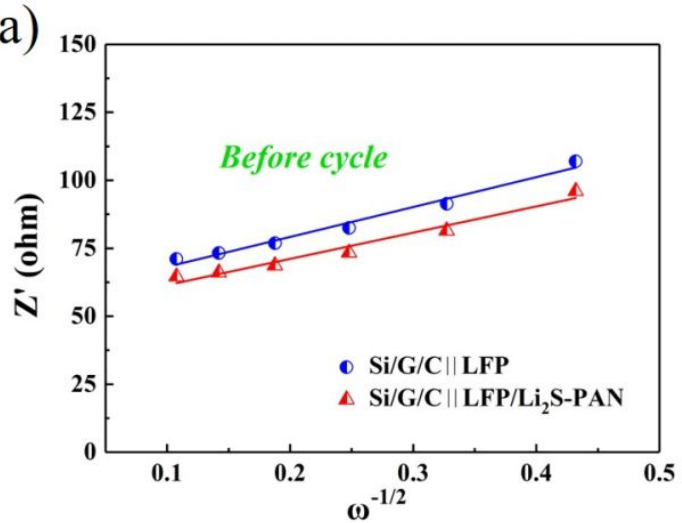

(b)

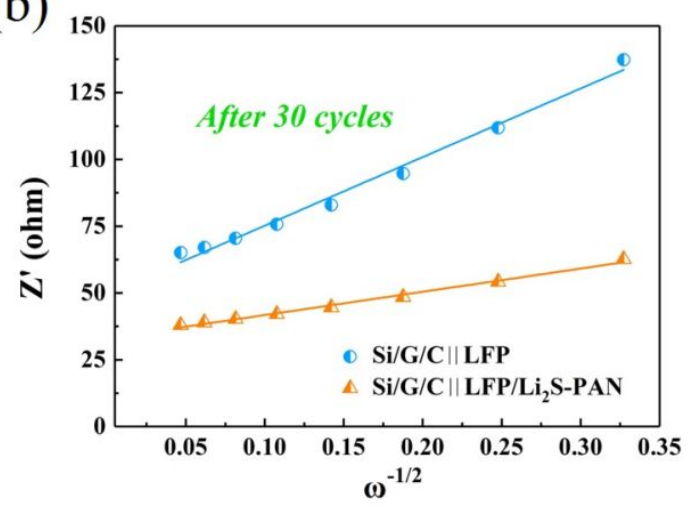

Figure S11. Z'- $\omega^{-1 / 2}$ curves of $\mathrm{Si} / \mathrm{G} / \mathrm{C} \| \mathrm{LiFePO}_{4}$ full-cell and $\mathrm{Si} / \mathrm{G} / \mathrm{C} \| \mathrm{LiFePO} / \mathrm{Li}_{2} \mathrm{~S}-\mathrm{PAN}$ full-cell (a) before and (b) after 30 cycles.

Table S2. The detailed anode SEI contents calculated based on XPS integral areas in Fig. 6 .

\begin{tabular}{|c|c|c|c|c|c|}
\hline $\begin{array}{c}\text { XPS } \\
\text { spectrum }\end{array}$ & $\begin{array}{c}\text { Chemical } \\
\text { bonds }\end{array}$ & $\begin{array}{c}\text { Cycled anode in } \\
\text { Si/G/C ||LFP } \\
\text { (Integral area) }\end{array}$ & $\begin{array}{c}\text { Cycled anode in } \\
\text { Si/G/C }|| L F P / L i_{2} S-P A N \\
\text { (Integral area) }\end{array}$ & $\begin{array}{c}\text { Cycled anode } \\
\text { in Si/G/C } \| \text { LFP } \\
\text { (\%) }\end{array}$ & $\begin{array}{c}\text { Cycled anode in } \\
\text { Si/G/C||LFP/Li } i_{2} S-P A N \\
(\%)\end{array}$ \\
\hline \multirow{6}{*}{$\mathrm{C}_{1 \mathrm{~s}}$} & $\mathrm{C}-\mathrm{C}$ & 45560.0 & 57799.2 & 44.2 & 46.1 \\
\hline & $\mathrm{C}-\mathrm{H}$ & 10984.2 & 20157.7 & 10.7 & 16.1 \\
\hline & $\mathrm{C}-\mathrm{O}$ & 35989.3 & 30855.5 & 34.9 & 24.6 \\
\hline & C-F & 3506.6 & 5559.6 & 3.4 & 4.4 \\
\hline & $\mathrm{ROCO}_{2} \mathrm{Li}$ & 3791.2 & 9915.4 & 3.7 & 7.9 \\
\hline & $\mathrm{LiCO}_{3}$ & 3295.7 & 1182.4 & 3.2 & 0.9 \\
\hline \multirow{5}{*}{$\mathbf{O}_{1 \mathrm{~s}}$} & $\mathrm{C}=\mathrm{O}$ & 46560.7 & 50276.6 & 17.4 & 31.9 \\
\hline & $\mathrm{LiOH}$ & 167174.7 & 75936.8 & 62.4 & 48.2 \\
\hline & $\mathrm{Li}_{2} \mathrm{CO}_{3}$ & 8099.7 & 1280.2 & 3.0 & 0.8 \\
\hline & $\mathrm{C}-\mathrm{OH}$ & 45840.1 & 25806.5 & 17.1 & 16.4 \\
\hline & $\mathrm{C}-\mathrm{O}$ & 50.7 & 4326.2 & 0.02 & 2.7 \\
\hline \multirow{2}{*}{$\mathbf{F}_{1 \mathrm{~s}}$} & $\mathrm{LiF}$ & 218652.7 & 214967.4 & 78.5 & 81.5 \\
\hline & $\mathrm{Li}_{\mathrm{x}} \mathrm{PO}_{\mathrm{y}} \mathrm{F}_{\mathrm{z}}$ & 59714.9 & 48739.9 & 21.5 & 18.5 \\
\hline
\end{tabular}




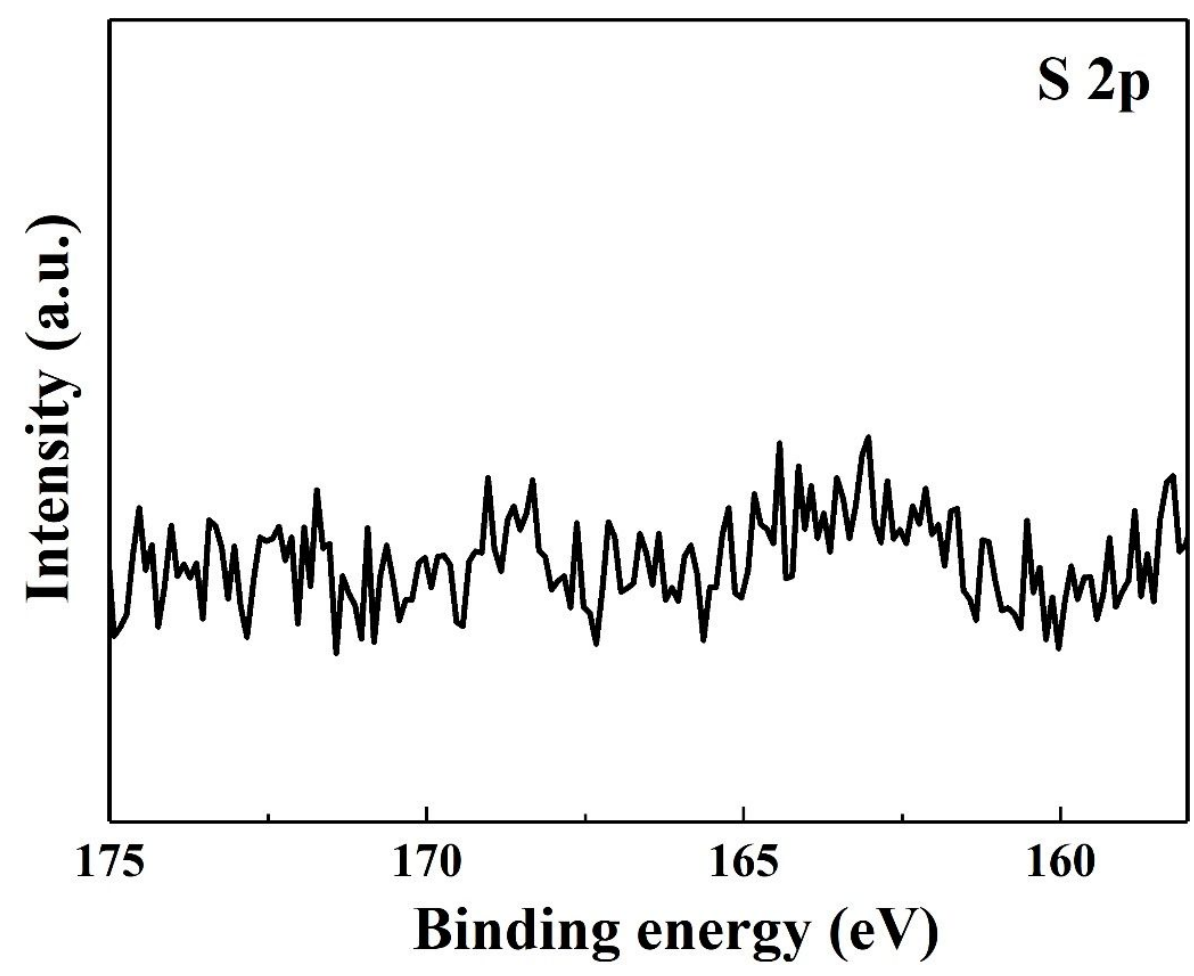

Figure S12. S 2p XPS spectra of the anode after the cycle of pre-lithiation full-cell.

\section{Reference}

[1] Zhang L.; Dose W.M.; Vu A.D.; Johnson C.S.; Lu W., Mitigating the Initial Capacity Loss and Improving the Cycling Stability of Silicon Monoxide using $\mathrm{Li}_{5} \mathrm{FeO}_{4}, J$ Power Sources 2018, 400, 549-555.

[2] Du J.; Wang W.; Sheng Eng A.Y.; Liu X.; Wan M.; She Z.W.; Sun Y., Metal/LiF/Li ${ }_{2} \mathrm{O}$ Nanocomposite for Battery Cathode Prelithiation: Trade-off between Capacity and Stability, Nano Letter 2020, 20, 546-552.

[3] Bie Y.; Yang J.; Wang J.; Zhou J.; Nuli Y., $\mathrm{Li}_{2} \mathrm{O}_{2}$ as a Cathode Additive for the Initial Anode Irreversibility Compensation in Lithium-ion Batteries, Chemical Communications 2017, 53, 8324-8327.

[4] Sun Y.; Li Y.; Sun J.; Li Y.; Pei A.; Cui Y., Stabilized $\mathrm{Li}_{3} \mathrm{~N}$ for Efficient Battery Cathode Prelithiation, Energy Storage Materials 2017, 6, 119-124.

[5] Sun Y.; Lee H.; She Z.W.; Liu N.; Sun J.; Li Y.; Cui Y., High-capacity Battery Cathode Prelithiation to Offset Initial Lithium Loss, Nature Energy 2016, 1, 15008. 
[6] Wang D.; Zhang Z.; Hong B.; Lai Y., Self-Sacrificial Organic Lithium Salt Enhanced Initial Coulombic Efficiency for Safer and Greener Lithium-Ion Batteries, Chemical Communications 2019, 55, 10737-10739. 